\title{
NONCONSERVATIVE STABILITY OF VISCOELASTIC PLATES SUBJECT TO TRIANGULARLY DISTRIBUTED FOLLOWER LOADS
}

\author{
Mouafo T.A. Robinson \\ University of KwaZulu-Natal, Discipline of Mechanical Engineering, Durban, South Africa, and \\ University of Dschang, Department of Physics, Cameroon; e-mail: armandmouafo@yahoo.fr \\ SARP ADALI \\ University of KwaZulu-Natal, Discipline of Mechanical Engineering, Durban, South Africa; e-mail: adali@ukzn.ac.za
}

\begin{abstract}
Divergence and flutter instabilities of viscoelastic rectangular plates under triangularly distributed tangential follower loads are studied. Two sets of boundary conditions are considered, namely, simply supported plates and plates with a combination of clamped and simply supported edges. The constitutive relations for the viscoelastic plates are of Kelvin-Voigt type with the effect of viscoelasticity on stability studied numerically. The method of solution is differential quadrature which is employed to discretize the equation of motion and the boundary conditions leading to a generalized eigenvalue problem. After verifying the method of solution, numerical results are given for the real and imaginary parts of the eigenfrequencies to investigate flutter and divergence characteristics and dynamic stability of the plates with respect to various problem parameters.
\end{abstract}

Keywords: viscoelastic plates, dynamic stability, triangularly distributed follower load

\section{Introduction}

Dynamic stability of elastic structures subject to nonconservative loads is of practical importance in such fields as aerospace, mechanical and civil engineering. As a result, the subject has been studied extensively to quantify the behaviour of beams, plates and shells under follower forces. These forces can be concentrated, uniformly distributed or triangularly distributed depending on the specific application. They act in the tangential direction and are not derivable from a potential due to their nonconservative nature as presented in works by Kumar and Srivasta (1986), Przybylski (1999), Gajewski (2000), Krillov (2013).

Early work on the nonconservative instability under uniformly distributed follower loads mostly involved one dimensional elastic structures, namely, columns (Sugiyama and Kawagoe, 1975; Leipholz, 1975; Chen and Ku, 1991). Stability of columns under triangularly distributed loads was studied by Leipholz and Bhalla (1977), Sugiyama and Mladenov (1983) and Ryu et al. (2000). More recent studies on nonconservative loading include columns subject to uniformly distributed follower loads by Kim (2010), Kim et al. (2008) and Kazemi-Lari et al. (2013) and to triangularly distributed follower loads by Kim (2011). The follower force can also be realized by means of properly shaped loading heads which can affect the displacements of the loaded end as studied by Tomski and Szmidla (2004) and Tomski and Uzny (2013b). The installation of Tomski's head can lead to a loading force which is tangent to the end of the column and can be conservative (Tomski and Uzny, 2008, 2013a). The force in this case is directed to a constant point which becomes a pole for the loading. Introduction of Tomski's head can lead to new characteristic curves such as pseudo flutter and allows one to control the dynamic behavior of the system. Studies on nonconservative stability of two-dimensional structures mostly involved rectangular plates under follower loads (Culkowski and Reismann, 1977; Farshad, 1978; Adali, 
1982) and under uniformly distributed tangential loads (Leipholz, 1978; Leipholz and Pfendt, 1982, 1983; Wang and Ji, 1992).

Recent research on the stability of elastic plates under nonconservative loads includes works by Zuo and Shreyer (1996), Kim and Park (1998), Kim and Kim (2000), and Jayaraman and Struthers (2005). Dynamic stability of functionally graded plates under uniformly distributed axial loads was studied by Ruan et al. (2012) and shells by Torki et al. (2014a,b). These studies neglected the effect of viscoelasticity on the stability of the columns and plates.

Dynamic stability of one-dimensional viscoelastic structures was the subject of the works by Marzani and Potapov (1999), Langthjem and Sugiyama (2000), Darabseh and Genin (2004), Zhuo and Fen (2005), Ilyasov and Ilyasova (2006) and Elfelsoufi and Azrar (2006). Recently, the dynamic stability of viscoelastic plates has been studied for a number of cases (Ilyasov and Aköz, 2000; Wang et al., 2007, 2009, 2013; Zhou and Wang, 2014; Robinson and Adali, 2016). Vibrations of a simply supported plate with nonlinear strain-displacement relations and subject to a uniformly distributed tangential force were studied by Robinson (2013). Dynamic stability of viscoelastic shells was studied by Ilyasov (2010).

Although the dynamic stability under triangularly distributed tangential forces have been studied in the case of columns (see Leipholz and Bhalla, 1977; Sugiyama and Mladenov, 1983; Ryu et al., 2000; Kim, 2011), dynamic stability of plates and, in particular, viscoelastic plates under this type of loading does not seem to be studied so far.

The present work extends the results of Robinson and Adali (2016) who studied nonconservative stability of viscoelastic plates with free edges and under uniformly distributed follower loads, to the case of plates with simply supported and simply supported-clamped plates and subject to triangularly distributed follower loads. Comparisons are given for the uniformly and triangularly distributed follower loads. The stability problem is solved for the simply supported plates and for plates with a combination of simple and clamped supports by the differential quadrature method. Divergence and flutter loads are determined and the effect of viscoelasticity and the boundary conditions on dynamic stability is investigated. The method of solution is verified against the known results in the literature.

\section{Viscoelastic plate subject to triangularly distributed load}

We consider a rectangular plate of uniform thickness $h$ having dimensions $a \times b$ in the $x$ and $y$ directions, respectively (see Fig. 1). It is subject to a non-uniform tangential follower force $q_{t}=q_{0} a(1-x / a)$.

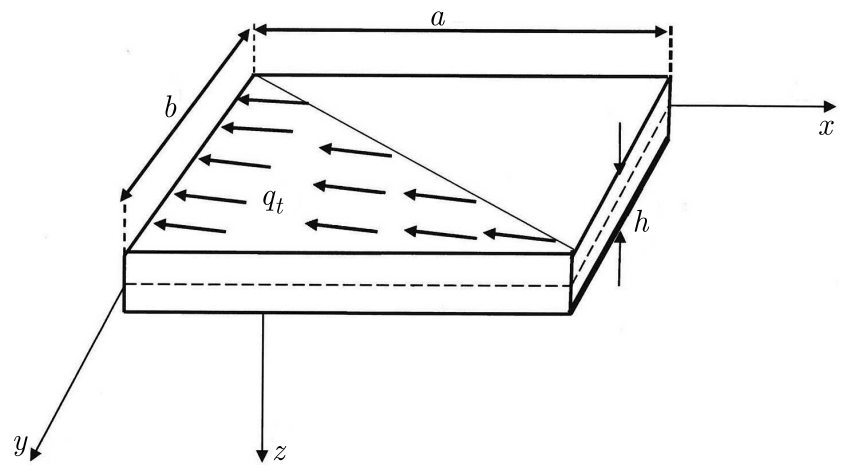

Fig. 1. Viscoelastic plate subjected to a triangular follower load

The material of the plate is viscoelastic which is expressed by the Kelvin-Voigt constitutive relations given by 


$$
\mathbf{s}_{i j}=2 G \mathbf{e}_{i j}+2 \eta \dot{\mathbf{e}}_{i j} \quad \boldsymbol{\sigma}_{i i}=3 K \varepsilon_{i i}
$$

where $\mathbf{s}_{i j}$ and $\mathbf{e}_{i j}$ are deviatoric tensors of stress and strain, respectively, and $\boldsymbol{\sigma}_{i i}$ and $\boldsymbol{\varepsilon}_{i i}$ are spherical tensors of stress and strain with $\eta$ denoting the viscoelastic coefficient. The bulk modulus $K$ and shear modulus $G$ can be expressed in terms of Young's modulus $E$ and Poisson's ratio $\nu$ as $K=E / 3(1-2 \nu)$ and $G=E /(1+2 \nu)$. The equation of vibration of the viscoelastic plate subject to a triangular follower load is first obtained in the Laplace domain (see Wang et al., 2007; Zhou and Wang, 2014). By the inverse Laplace transformation, the governing equation can be expressed in the time domain as

$$
\frac{h^{3}}{12}\left(A_{3}+A_{4} \frac{\partial}{\partial t}+A_{5} \frac{\partial^{2}}{\partial t^{2}}\right) \nabla^{4} w+\left(A_{1}+A_{2} \frac{\partial}{\partial t}\right)\left(\frac{q_{0}(a-x)^{2}}{2} \frac{\partial^{2} w}{\partial x^{2}}+\rho h \frac{\partial^{2} w}{\partial t^{2}}\right)=0
$$

where $\rho$ is density of the plate and

$$
\begin{array}{lll}
A_{1}=3 K+4 G & A_{1}=3 K+4 G & A_{2}=4 \eta \\
A_{3}=4 G(3 K+G) & A_{4}=\eta(8 G+12 K) & A_{5}=4 \eta^{2}
\end{array}
$$

and

$$
\nabla^{4} w=\frac{\partial^{4} w}{\partial x^{4}}+2 \frac{\partial^{4} w}{\partial x^{2} \partial y^{2}}+\frac{\partial^{4} w}{\partial y^{4}}
$$

After introducing dimensionless coefficients

$$
X=\frac{x}{a} \quad Y=\frac{y}{b} \quad \bar{w}=\frac{w}{h} \quad \lambda=\frac{a}{b}
$$

and

$$
q=\frac{6 q_{0} a^{4}\left(1-\nu^{2}\right)}{E h^{3}} \quad \tau=\frac{t h}{a^{2}} \sqrt{\frac{E}{12 \rho\left(1-\nu^{2}\right)}} \quad H=\frac{\eta h}{a^{2}} \sqrt{\frac{1}{12 \rho\left(1-\nu^{2}\right) E}}
$$

the non-dimensional equation of motion is obtained as

$$
\left(1+g_{1} \frac{\partial}{\partial \tau}+g_{2} \frac{\partial^{2}}{\partial \tau^{2}}\right) \nabla^{4} \bar{w}+\left(1+g_{3} \frac{\partial}{\partial \tau}\right)\left(q(1-X)^{2} \frac{\partial^{2} \bar{w}}{\partial X^{2}}+\frac{\partial^{2} \bar{w}}{\partial \tau^{2}}\right)=0
$$

where

$$
\begin{aligned}
& g_{2}=\frac{4(1-2 \nu)(1+\nu)^{2}}{3} H^{2} \quad g_{3}=\frac{4(1-2 \nu)(1+\nu)}{3(1-\nu)} H \\
& \nabla^{4} \bar{w}=\frac{\partial^{4} \bar{w}}{\partial X^{4}}+2 \lambda^{2} \frac{\partial^{4} \bar{w}}{\partial X^{2} \partial Y^{2}}+\lambda^{4} \frac{\partial^{4} \bar{w}}{\partial Y^{4}}
\end{aligned}
$$

In equations (2.8), $H$ is the dimensionless delay time of the material and $\tau$ is the dimensionless time defined in Eq. (2.6). Let

$$
\bar{w}(X, Y, \tau)=W(X, Y) \mathrm{e}^{\mathrm{j} \omega \tau}
$$

where $\mathrm{j}=\sqrt{-1}$ and $\omega$ the dimensionless vibration frequency. Substitution of equation (2.9) into equation (2.7) yields the differential equation

$$
\left(1+g_{1} j \omega+g_{2} \mathrm{j}^{2} \omega^{2}\right) \nabla^{4} W+\left(1+g_{3} \mathrm{j} \omega\right)\left(q(1-X)^{2} \frac{\partial^{2} W}{\partial X^{2}}+\mathrm{j}^{2} \omega^{2}\right)=0
$$

in terms of the space variables $X$ and $Y$. The boundary conditions considered in the present work are the simply supported plates (SSSS) and plates with two opposite edges clamped and two others simply supported (CSCS). 
SSSS boundary conditions are given by

$$
\bar{w}(X, Y, \tau)= \begin{cases}\left.\frac{\partial^{2} \bar{w}}{\partial X^{2}}\right|_{X=0,1}=0 & \text { for } \quad 0 \leqslant Y \leqslant 1 \\ \left.\frac{\partial^{2} \bar{w}}{\partial Y^{2}}\right|_{Y=0,1}=0 & \text { for } \quad 0 \leqslant X \leqslant 1\end{cases}
$$

CSCS boundary conditions are given by

$$
\bar{w}(X, Y, \tau)= \begin{cases}\left.\frac{\partial \bar{w}}{\partial X}\right|_{X=0,1}=0 & \text { for } \quad 0 \leqslant Y \leqslant 1 \\ \left.\frac{\partial^{2} \bar{w}}{\partial Y^{2}}\right|_{Y=0,1}=0 & \text { for } \quad 0 \leqslant X \leqslant 1\end{cases}
$$

\section{Differential quadrature (DQ) method}

The DQ method involves approximating the partial derivatives of the function $W(X, Y)$ at a sample point $\left(X_{i}, Y_{j}\right)$ by the weighted sum of the function $W_{i j}$ (see Bert and Malik, 1996; Krowiak, 2008). Let the number of sample points denoted by $N$ in the $X$ direction and $M$ in the $Y$ direction. The $r$-th order partial derivative with respect to $X, s$-th order partial derivative with respect to $Y$ and the $(r+s)$-th order mixed partial derivative of $W(X, Y)$ with respect to both $X$ and $Y$ are discretely expressed at the point $\left(X_{i}, Y_{j}\right)$ as

$$
\begin{aligned}
& \frac{\partial^{r} W\left(X_{i}, Y_{j}\right)}{\partial X^{r}}=\sum_{k=1}^{N} A_{i k}^{(r)} W_{k j} \quad \frac{\partial^{s} W\left(X_{i}, Y_{j}\right)}{\partial Y^{s}}=\sum_{k=1}^{M} B_{j l}^{(s)} W_{i l} \\
& \frac{\partial^{r+s} W\left(X_{i}, Y_{j}\right)}{\partial X^{r} \partial Y^{s}}=\sum_{k=1}^{N} A_{i k}^{(r)} \sum_{l=1}^{M} B_{j l}^{(s)} W_{k l}
\end{aligned}
$$

where $i=1,2, \ldots, N, k=1,2, \ldots, N-1, j=1,2, \ldots, M$ and $l=1,2, \ldots, M-1$.

For $r=s=1$, the coefficients $A_{i k}^{(r)}$ and $B_{j l}^{(s)}$ are defined as

$$
\begin{aligned}
A_{i k}^{(1)} & =\left\{\begin{array}{lr}
\prod_{\mu=1, \mu \neq i}^{N} \frac{X_{i}-X_{\mu}}{\left(X_{i}-X_{k}\right) \prod_{\mu-1, \mu \neq k}^{N}\left(X_{k}-X_{\mu}\right)} & \text { for } \quad i, k=1,2, \ldots, N \quad(i \neq k) \\
\sum_{\mu-1, \mu \neq k}^{N} \frac{1}{X_{i}-X_{\mu}} & \text { for } \quad i=1,2, \ldots, N \quad(i=k)
\end{array}\right. \\
B_{j l}^{(1)} & = \begin{cases}\prod_{\mu=1, \mu \neq j}^{M} \frac{Y_{j}-Y_{\mu}}{\left(Y_{j}-Y_{l}\right) \prod_{\mu-1, \mu \neq l}^{M}\left(Y_{l}-Y_{\mu}\right)} & \text { for } \quad j, l=1,2, \ldots, M \quad(j \neq l) \\
\sum_{\mu=1, \mu \neq j}^{M} \frac{1}{Y_{j}-Y_{\mu}} & \text { for } \quad j=1,2, \ldots, M \quad(j=l)\end{cases}
\end{aligned}
$$


For $r=2,3, \ldots, N-1$ and $s=2,3, \ldots, M-1$

$$
\begin{aligned}
& A_{i k}^{(r)}= \begin{cases}r\left(A_{i i}^{(r-1)} A_{i k}^{(1)}-\frac{A_{i k}^{(r-1)}}{X_{i}-X_{k}}\right) & \text { for } \quad i, k=1,2, \ldots, N \quad(i \neq k) \\
-\sum_{\mu=1, \mu \neq i}^{N} A_{i \mu}^{(r)} & \text { for } \quad i=1,2, \ldots, N \quad(i=k)\end{cases} \\
& B_{j l}^{(s)}= \begin{cases}s\left(B_{j j}^{(s-1)} B_{j l}^{(1)}-\frac{B_{j l}^{(s-1)}}{Y_{j}-Y_{l}}\right) & \text { for } \quad j, l=1,2, \ldots, M \quad(j \neq l) \\
-\sum_{\mu=1, \mu \neq j}^{M} B_{j \mu}^{(s)} & \text { for } \quad j=1,2, \ldots, M \quad(j=l)\end{cases}
\end{aligned}
$$

The distribution of the grid points are taken as non-uniform, and for the simply supported plate, the grid points are specified as

$$
\begin{aligned}
& X_{1}=0 \quad X_{N}=1 \quad X_{i}=\frac{1}{2}\left[1-\cos \left(\frac{2 i-3}{2 N-4} \pi\right)\right] \quad \text { for } \quad i=2,3, \ldots, N-1 \\
& Y_{1}=0 \quad Y_{M}=1 \quad Y_{j}=\frac{1}{2}\left[1-\cos \left(\frac{2 j-3}{2 N-4} \pi\right)\right] \quad \text { for } \quad j=2,3, \ldots, M-1
\end{aligned}
$$

For the plate with two opposite edges simply supported and other two edges clamped, the $\delta$ method combined with the weighted coefficient method is adopted. Thus, the grid points for the CSCS plate are given by

$$
\begin{array}{lcccc}
X_{1}=0 & X_{2}=\delta & X_{N-1}=1-\delta \quad X_{N}=1 & \\
X_{i}=\frac{1}{2}\left[1-\cos \left(\frac{i-2}{N-3} \pi\right)\right] & \text { for } i=3,4, \ldots, N-2 & \\
Y_{1}=0 & Y_{M}=1 & Y_{j}=\frac{1}{2}\left[1-\cos \left(\frac{2 j-3}{2 N-4} \pi\right)\right] \quad \text { for } j=2,3, \ldots, M-1
\end{array}
$$

where $\delta \ll 1$. Using equation (3.1), the discretized form of differential equation (2.10) can be expressed as

$$
\begin{aligned}
& c_{1} \mathrm{j}^{3} W_{i j} \omega^{3}+\left(c_{2} S_{i j}+W_{i j}\right) \mathrm{j}^{2} \omega^{2}+\left(c_{3} S_{i j}\right. \\
& \left.\quad+c_{1} q(1-X)^{2} \sum_{k=1}^{N} A_{i k}^{(2)} W_{k j}\right) \mathrm{j} \omega+q(1-X)^{2} \sum_{k=1}^{N} A_{i k}^{(2)} W_{k j}=0
\end{aligned}
$$

where

$$
\begin{aligned}
& S_{i j}=\sum_{k=1}^{N} A_{i k}^{(4)} W_{k j}+2 \lambda^{2} \sum_{l=1}^{M} B_{j l}^{(2)} \sum_{k=1}^{N} A_{i k}^{(2)} W_{k l}+\lambda^{4} \sum_{l=1}^{M} B_{j l}^{(4)} W_{i l} \\
& c_{1}=\frac{4(1-2 \nu)(1+\nu)}{3(1-\nu)} H \quad c_{2}=\frac{4(1-2 \nu)(1+\nu)^{2}}{3} H^{2} \quad c_{3}=\frac{4(1-2 \nu)(1+\nu)}{3} H
\end{aligned}
$$

The discretized form of boundary conditions (2.11) are given by

$$
\begin{aligned}
& W_{1 j}=W_{N j}=W_{i 1}=W_{i M}=0 \quad \text { for } \quad i=1,2, \ldots, N \wedge j=1,2, \ldots, M \\
& \sum_{k=1}^{N} A_{i k}^{(2)} W_{k j}=0 \quad \text { for } \quad i=1, N \wedge j=1,2, \ldots, M \\
& \sum_{l=1}^{M} B_{j l}^{(2)} W_{i l}=0 \quad \text { for } \quad i=1,2, \ldots, N \quad \wedge j=1, M
\end{aligned}
$$


The corresponding equations for boundary conditions (2.12) are

$$
\begin{aligned}
& W_{1 j}=W_{N j}=W_{i 1}=W_{i M}=0 \quad \text { for } \quad i=1,2, \ldots, N \wedge j=1,2, \ldots, M \\
& \sum_{k=1}^{N} A_{i k}^{(1)} W_{k j}=0 \quad \text { for } \quad i=1,2, \ldots, N-1 \wedge j=2,3, \ldots, M-2 \\
& \sum_{l=1}^{M} B_{j l}^{(2)} W_{i l}=0 \quad \text { for } \quad i=1,2, \ldots, N \quad \wedge j=1, M
\end{aligned}
$$

\section{Numerical results and discussion}

The results for the viscoelastic plate subject to a triangularly distributed tangential force are given in comparison to the results for a viscoelastic plate subject to a uniformly distributed tangential force which was studied in Wang et al. (2007) and Zhou and Wang (2014). The results for the SSSS and CSCS boundary conditions are given in Table 1 for $H=10^{-5}$ (nondimensional viscoelasticity coefficient). Table 1 shows that the flutter load, denoted by $q_{f}$, is higher in the case of the load having triangular distribution as expected. In Table $1, q_{d 1}$ and $q_{d 2}$ denote the divergence loads of the 1st and 2nd modes, respectively.

Table 1. Comparison of flutter loads $q$ of viscoelastic plates with $H=10^{-5}$ for various aspect ratios

\begin{tabular}{|c|c|c|c|}
\hline $\begin{array}{c}\text { Aspect } \\
\text { ratio } \lambda\end{array}$ & $\begin{array}{c}\text { Boundary } \\
\text { conditions }\end{array}$ & $\begin{array}{c}\text { Uniformly } \\
\text { distributed load, } \\
\text { Wang } \text { et al. }(2007)\end{array}$ & $\begin{array}{c}\text { Triangularly } \\
\text { distributed } \\
\text { load }\end{array}$ \\
\hline \hline \multirow{3}{*}{1.0} & SSSS & $\begin{array}{c}q_{d 1}=67.5 \\
q_{d 2}=132.1\end{array}$ & $\begin{array}{c}q_{d 1}=95.1 \\
q_{d 2}=225.1\end{array}$ \\
\cline { 2 - 4 } & CSCS & $\begin{array}{l}q_{d 1}=143.5 \\
q_{f}=168.0\end{array}$ & $q_{f}=226.0$ \\
\hline \multirow{3}{*}{1.5} & SSSS & $\begin{array}{l}q_{d 1}=136.8 \\
q_{d 2}=224.7\end{array}$ & $\begin{array}{c}q_{d 1}=174.0 \\
q_{d 2}=329.0\end{array}$ \\
\cline { 2 - 4 } & $\mathrm{CSCS}$ & $q_{f}=202.8$ & $q_{f}=270.0$ \\
\hline \multirow{3}{*}{2.0} & $\mathrm{SSSS}$ & $\begin{array}{l}q_{d 1}=224.8 \\
q_{d 2}=340.5\end{array}$ & $\begin{array}{c}q_{d 1}=273.04 \\
q_{d 2}=453.2\end{array}$ \\
\cline { 2 - 4 } & $\mathrm{CSCS}$ & $q_{f}=251.5$ & $q_{f}=333.0$ \\
\hline
\end{tabular}

Figures 2-4 show the real and the imaginary parts of the first three frequencies plotted against the load $q$ for uniformly and triangularly distributed tangential loads for the SSSS plates with $H=10^{-5}$ and $\lambda=1, \lambda=1.5$ and $\lambda=2$, respectively. The corresponding results for the imaginary part of the frequencies for $H=10^{-3}$ are given in Figs. 5 and 6 . It is noted that the results given in Figs. 2-6 for the uniformly distributed tangential load are the same as the ones given in Wang et al. (2007). As such, they provide the verification of the method of solution outlined in Section 3.

Comparisons of the loads with uniform and triangular distributions indicate that the results are qualitatively similar, but the magnitudes of the follower load causing divergence or flutter instability differ considerably. Comparisons between Figs. 2a, 3a, 4a $\left(H=10^{-5}\right)$ and Figs. 5a, $5 \mathrm{~b}$ and $6\left(H=10^{-3}\right)$ indicate that the imaginary parts of the frequencies remain positive for $H=10^{-3}$ up to the flutter load. The corresponding results for the CSCS plates with $H=10^{-5}$ are given in Figs. 7-9 with $\lambda=1, \lambda=1.5$ and $\lambda=2$, respectively. The results for the uniformly distributed tangential loads are also shown in the figures which verify the results of Wang et al. 

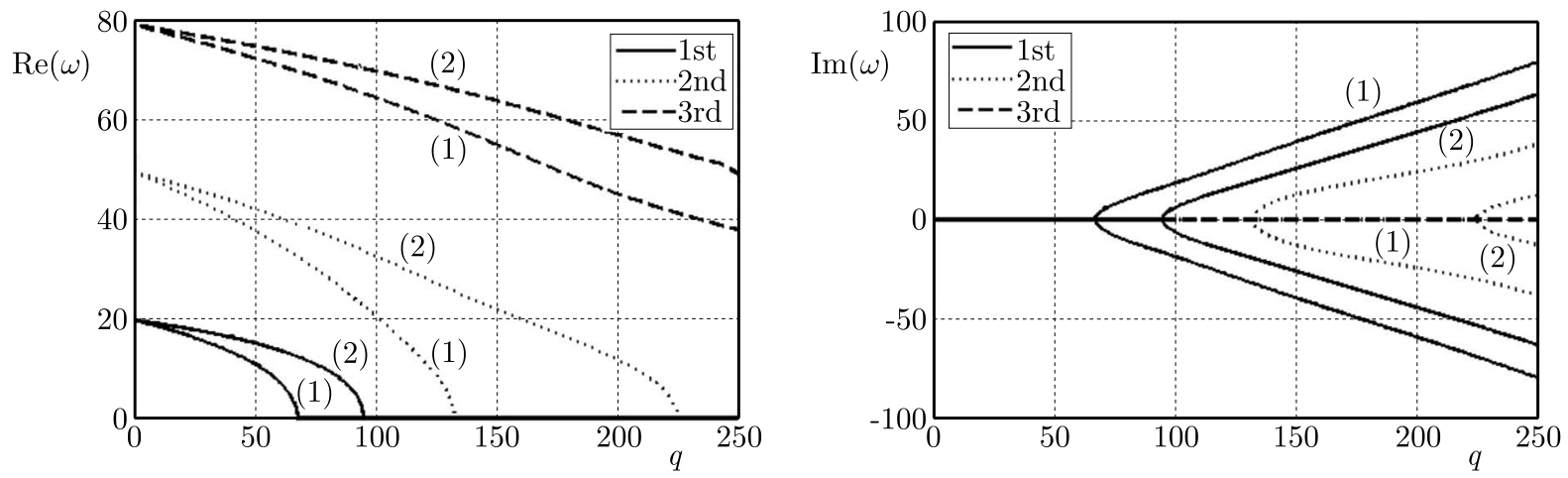

Fig. 2. First three frequencies of SSSS plate vs. follower force for $\lambda=1, H=10^{-5}$; (1) uniformly distributed load, (2) triangularly distributed load
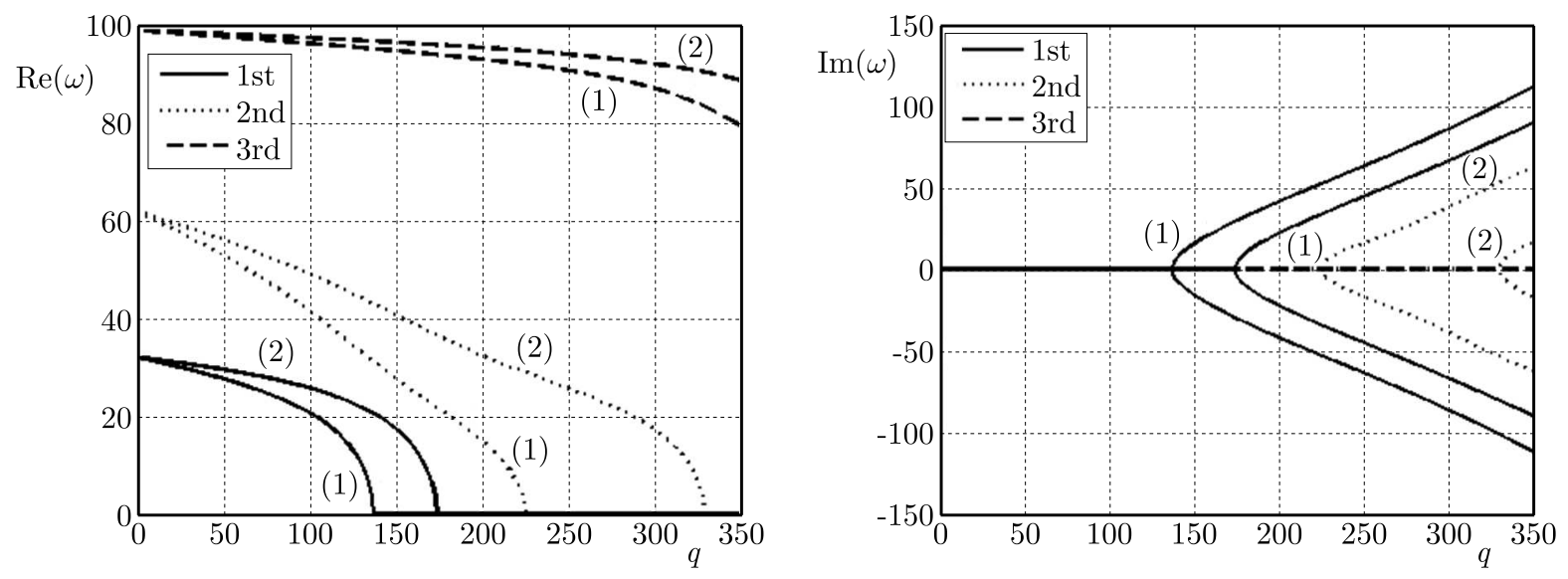

Fig. 3. First three frequencies of SSSS plate vs. follower force for $\lambda=1.5, H=10^{-5}$; (1) uniformly distributed load, (2) triangularly distributed load
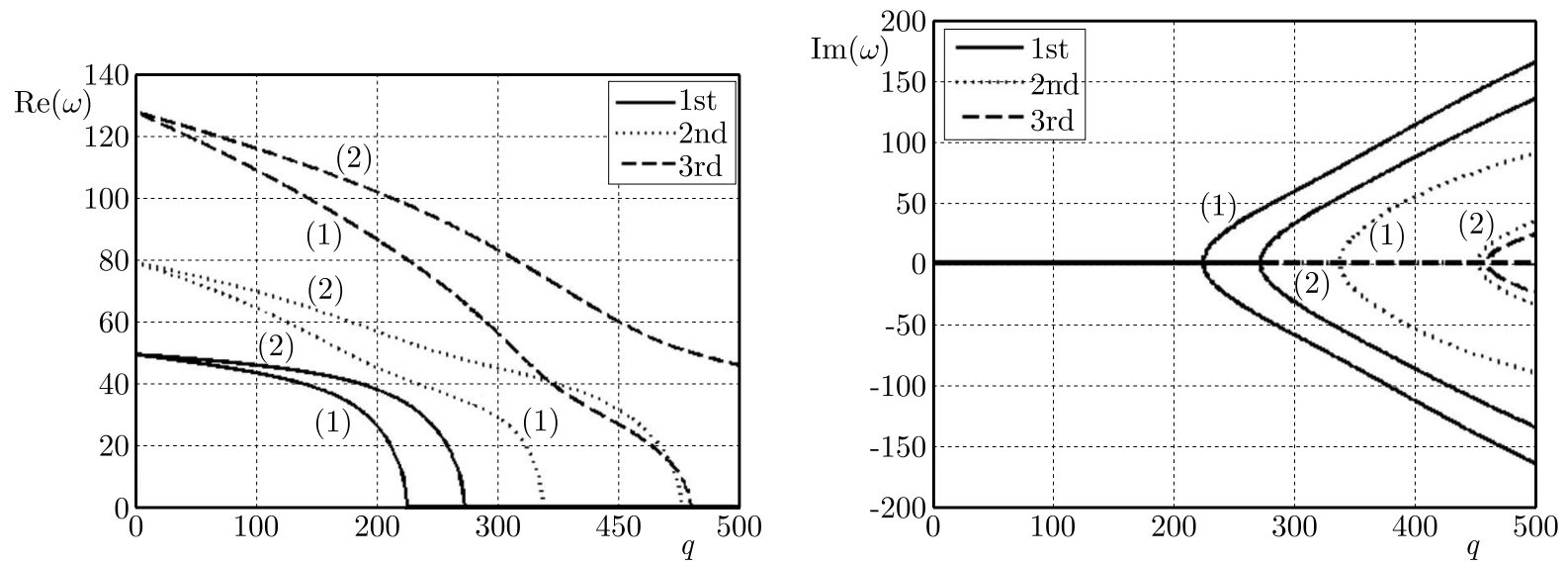

Fig. 4. First three frequencies of SSSS plate vs. follower force for $\lambda=2, H=10^{-5}$; (1) uniformly distributed load, (2) triangularly distributed load

(2007). In this case, it is observed that the real parts of the vibration modes behave differently as compared to the SSSS plates shown in Figs. 2-4. For the case $\lambda=1$ (Fig. 7a), the real parts of the first and the third modes join to form a single mode. For $\lambda=1.5$ and $\lambda=2$, the first and the second modes join as shown in Figs. 8a and 9a, respectively. Thus, in the case of CSCS boundary conditions, there exists a threshold value $q$ above which the first mode can join the second or third mode to form a single mode, and this value depends on the aspect ratio. Moreover, it is 

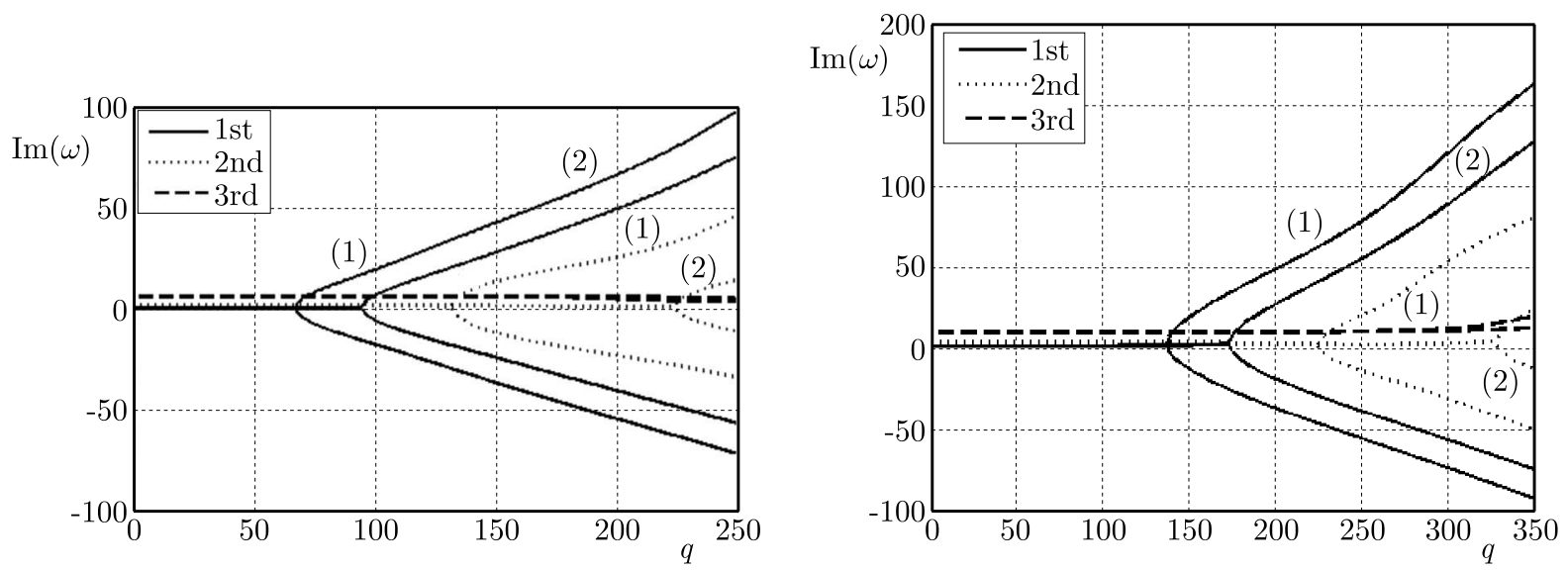

Fig. 5. Imaginary parts of frequencies of SSSS plate vs. follower force for (a) $\lambda=1$ and (b) $\lambda=1.5$, $H=10^{-3}$; (1) uniformly distributed load, (2) triangularly distributed load

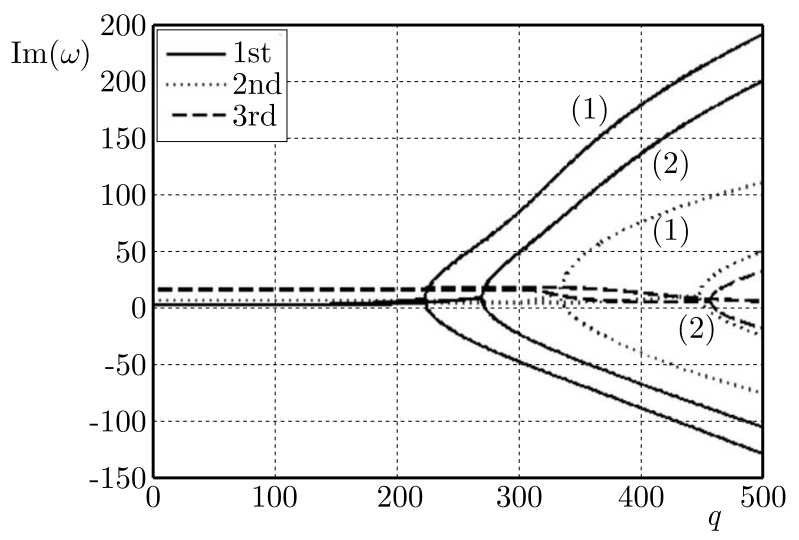

Fig. 6. Imaginary part of frequency of SSSS plate vs. follower force for $\lambda=2, H=10^{-3}$; (1) uniformly distributed load, (2) triangularly distributed load
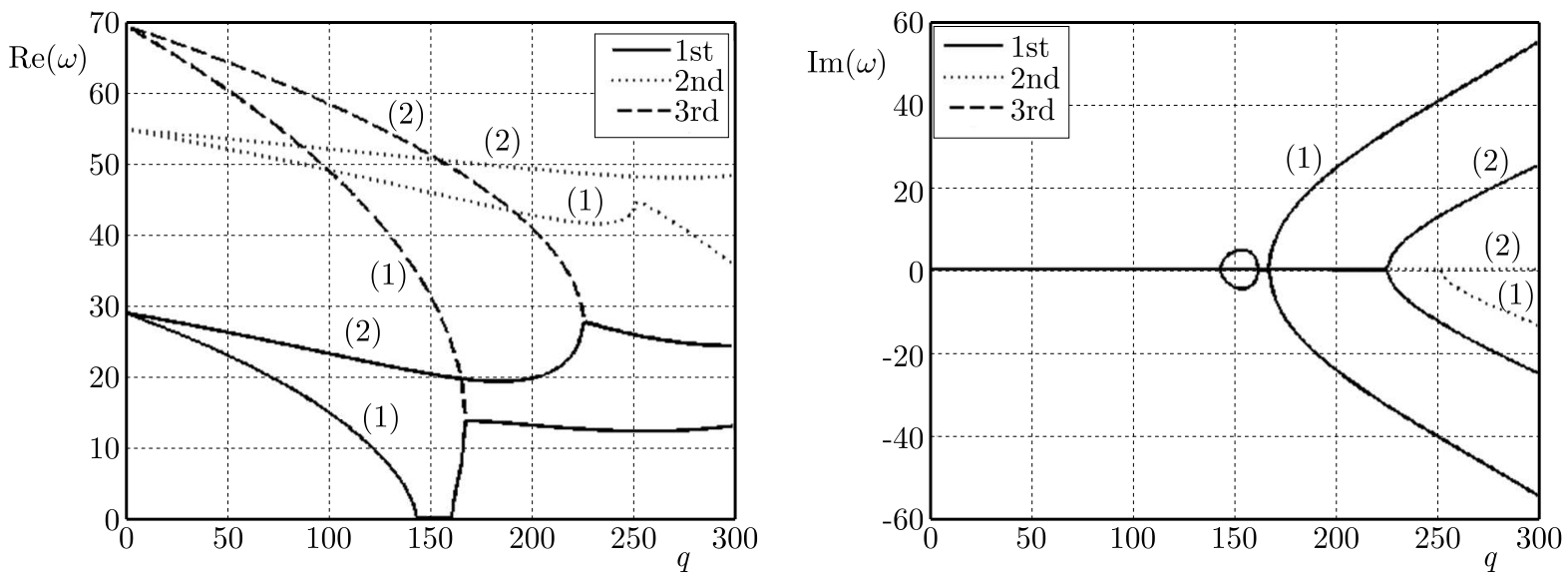

Fig. 7. First three frequencies of CSCS plate vs. follower force for $\lambda=1, H=10^{-5}$; (1) uniformly distributed load, (2) triangularly distributed load

observed that for the aspect ratios of $\lambda=1.5$ and $\lambda=2$, the plate does not show divergence instability and loses stability by flutter.

For the CSCS boundary conditions with $H=10^{-3}$, the results are given in Figs. 10-12. For this value of $H=10^{-3}$, the real parts of the frequencies do not form a single mode and the imaginary parts remain positive until the threshold values are exceeded and the flutter 

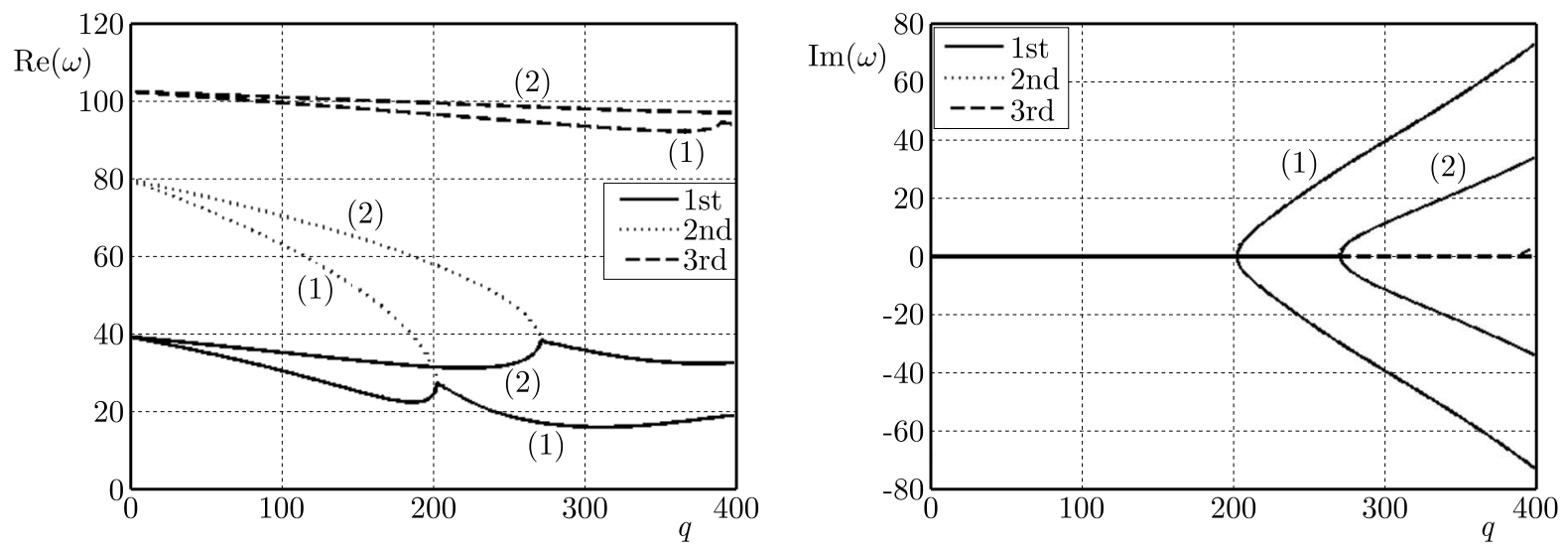

Fig. 8. First three frequencies of CSCS plate vs. follower force for $\lambda=1.5, H=10^{-5}$; (1) uniformly distributed load, (2) triangularly distributed load
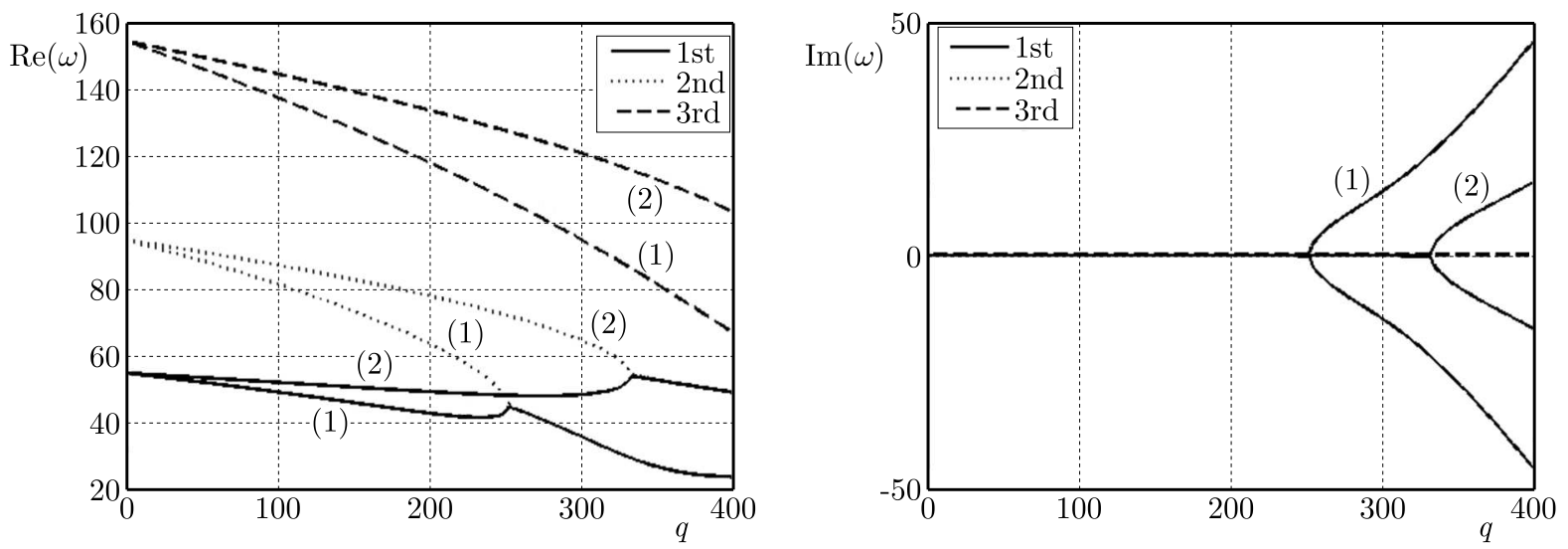

Fig. 9. First three frequencies of CSCS plate vs. follower force for $\lambda=2, H=10^{-5}$; (1) uniformly distributed load, (2) triangularly distributed load
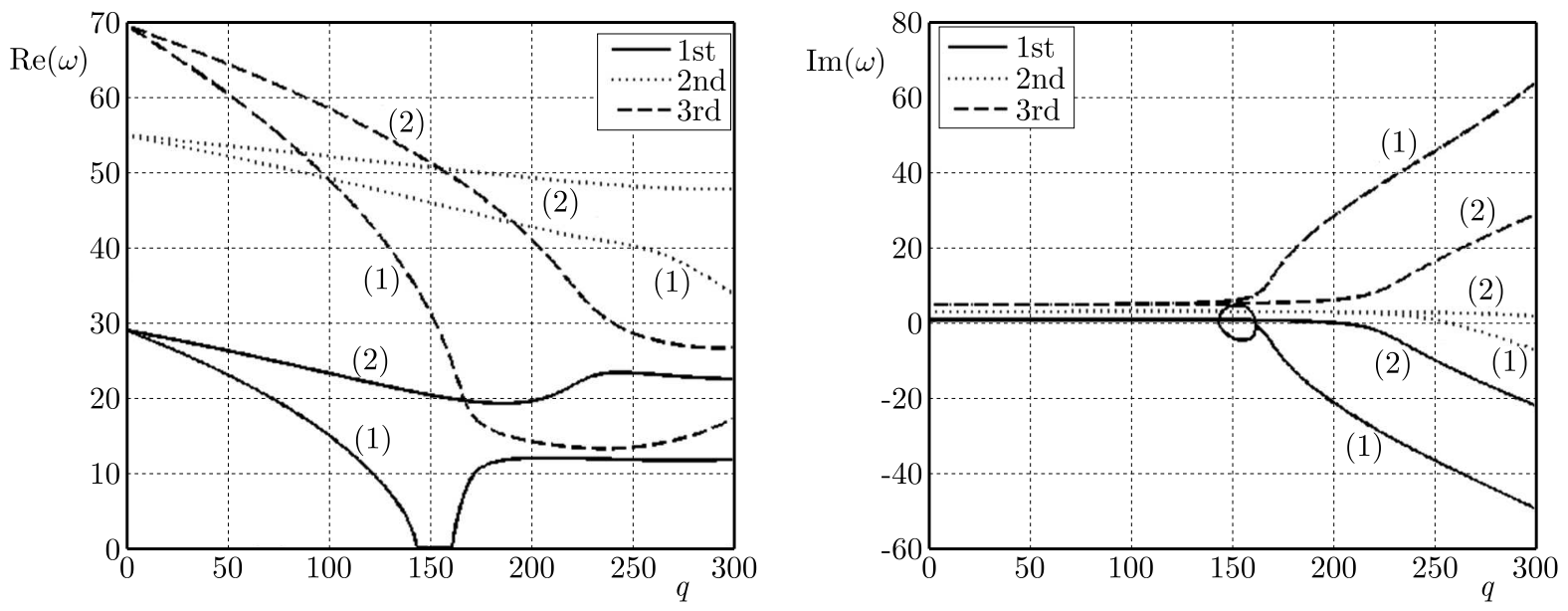

Fig. 10. First three frequencies of CSCS plate vs. follower force for $\lambda=1, H=10^{-3}$; (1) uniformly distributed load, (2) triangularly distributed load

instability occurs as shown in Figs. 10b, 11b and 12b. The imaginary parts of the frequencies exhibit negative values for $q \geqslant q_{f}$ leading to an exponential growth of the deflection. 

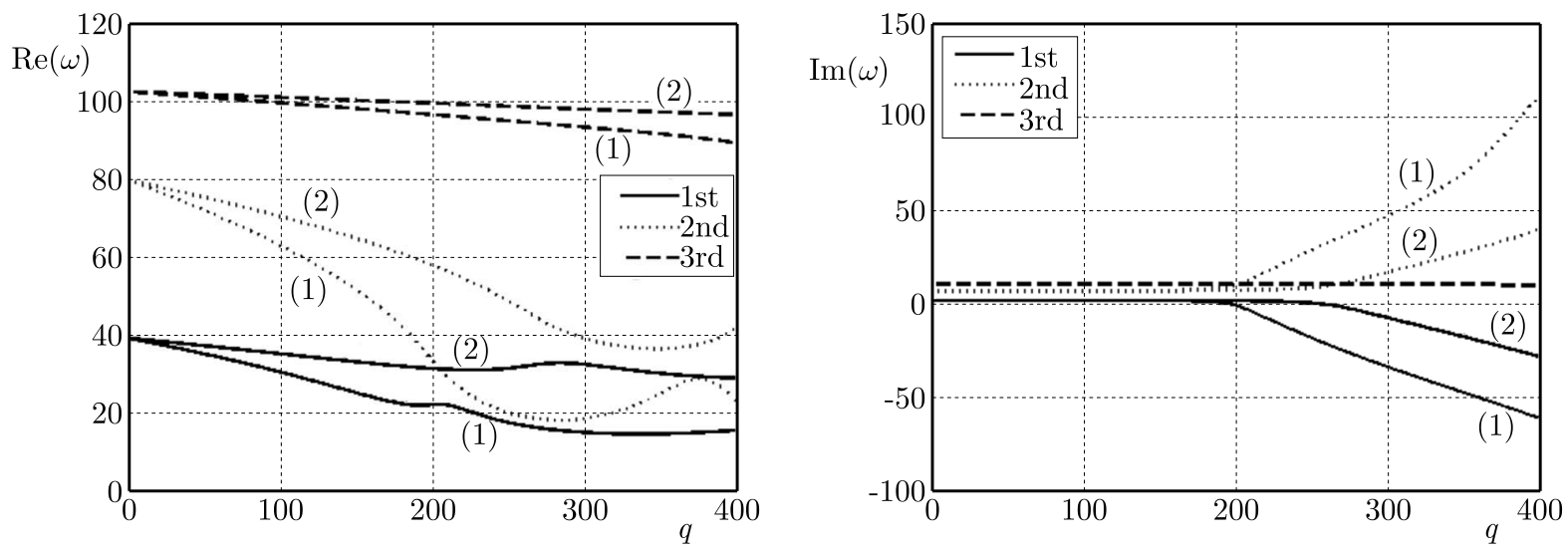

Fig. 11. First three frequencies of CSCS plate vs. follower force for $\lambda=1.5, H=10^{-3}$; (1) uniformly distributed load, (2) triangularly distributed load
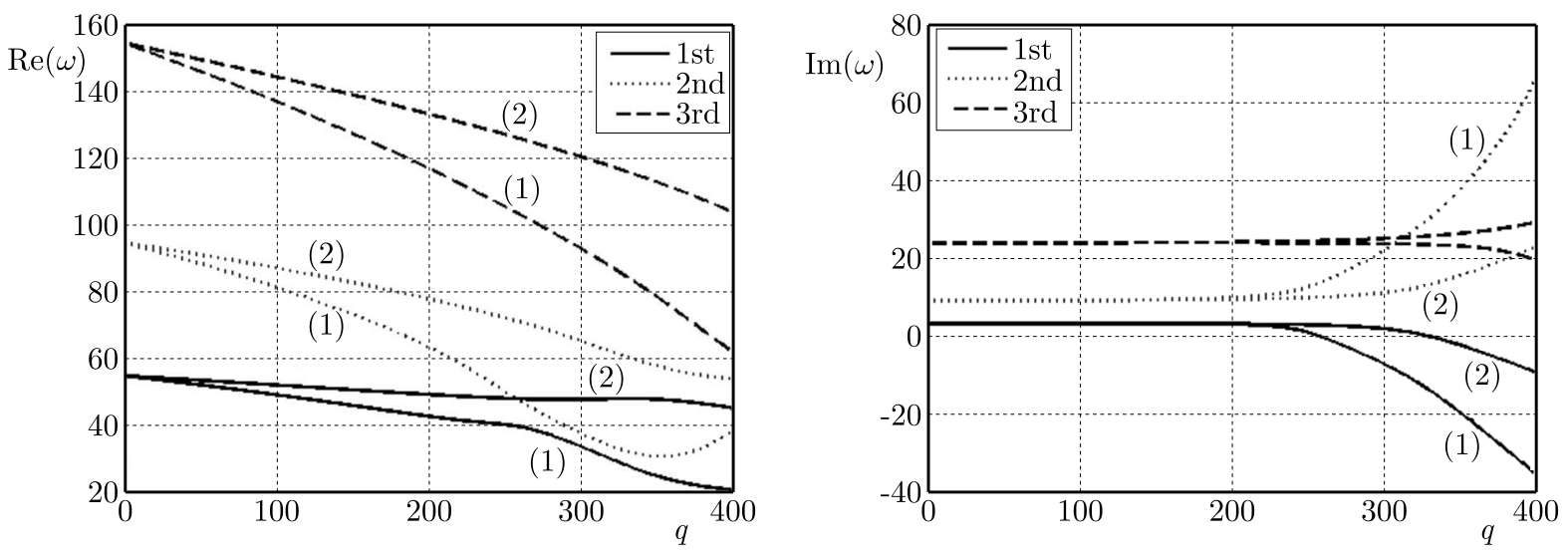

Fig. 12. First three frequencies of CSCS plate vs. follower force for $\lambda=2, H=10^{-3}$; (1) uniformly distributed load, (2) triangularly distributed load

\section{Conclusions}

The differential quadrature method is employed to study the dynamic stability of rectangular viscoelastic plates subject to triangularly distributed tangential follower loads. The Kelvin-Voigt viscoelastic model is taken as the constitutive equation of the plate. Two boundary conditions are investigated, namely, simple supports and a combination of simple and fixed supports. The solution is verified against the previous results obtained for SSSS and CSCS viscoelastic plates subject to uniformly distributed tangential loads by Wang et al. (2007).

Numerical results are given to study the effects of the aspect ratio and degree of viscoelasticity on the real and imaginary parts of the frequencies. The effect of uniformly and triangularly distributed follower loads on dynamic stability is compared numerically. It is observed that in the case of CSCS plates, the flutter instability occurs before the divergence instability for higher aspect ratios. In the case of SSSS plates, the degree of viscoelasticity does not affect the divergence load, but this effect is more pronounced for CSCS plates. At higher levels of viscoelasticity (higher values of $H$ ), the imaginary parts of the complex frequencies become positive rather than zero for low values of the follower load. The results obtained for the present case can be extended to different follower load cases and, in particular, to the cases where the direction of the load is controlled by a head (Tomski and Uzny, 2013b). 


\section{Acknowledgment}

The research reported in this paper was supported by research grants from the University of KwaZulu-Natal (UKZN) and from National Research Foundation (NRF) of South Africa. The authors gratefully acknowledge the support provided by UKZN and NRF.

\section{References}

1. AdAli S., 1982, Stability of a rectangular plate under nonconservative and conservative forces, International Journal of Solids and Structures, 18, 1043-1052

2. Bert C.W., Malik M., 1996, Implementing multiple boundary conditions in the DQ solution of higher-order PDE's: Application to free vibration of plates, International Journal of Numerical Methods in Engineering, 39, 1237-1258

3. Chen L.-W., Ku D.-M., 1991, Stability analysis of a Timoshenko beam subjected to distributed follower forces using finite elements, Computers and Structures, 41, 813-819

4. Culkowski P.M., Reismann H., 1977, Plate buckling due to follower edge forces, ASME Journal of Applied Mechanics, 99, 768-769

5. Darabseh T.T., Genin J., 2004, Dynamic stability of viscoelastic columns loaded by a follower force, Journal of Mechanical Engineering Science, 218, 1091-1101

6. Elfelsoufi Z., Azrar L., 2006, Integral equation formulation and analysis of the dynamic stability of damped beams subjected to subtangential follower forces, Journal of Sound and Vibration, 296, $690-713$

7. FARSHAD M., 1978, Stability of cantilever plates subjected to biaxial subtangential loading, Journal of Sound and Vibration, 58, 555-561

8. Gajewski A., 2000, Vibrations and stability of a non-conservatively compressed prismatic column under nonlinear creep conditions, Journal of Theoretical and Applied Mechanics, 38, 259-270

9. Ilyasov M.H., 2010, Parametric vibrations and stability of viscoelastic shells, Mechanics of TimeDependent Materials, 14, 153-171

10. Ilyasov M.H., AkÖZ Y.A., 2000, The vibration and dynamic stability of viscoelastic plates, International Journal of Engineering Science, 38, 695-714

11. Ilyasov M.H., Ilyasova N.M., 2006, Flutter of viscoelastic strips, Mechanics of Time-Dependent Materials, 10, 201-213

12. Jayaraman G., Struthers A., 2005, Divergence and flutter instability of elastic specially orthotropic plates subject to follower forces, Journal of Sound and Vibration, 281, 357-373

13. Kazemi-Lari M.A., Ghavanloo E., Fazelzadeh S.A., 2013, Structural instability of carbon nanotubes embedded in viscoelastic medium and subjected to distributed tangential load, Journal of Mechanical Science and Technology, 27, 2085-2091

14. Kim J.H., Kim H.S., 2000, A study on the dynamic stability of plates under a follower load, Computers and Structures, 74, 351-363

15. Kim J.H., PARK J.H., 1998, On the dynamic stability of rectangular plates subjected to intermediate follower forces, Journal of Sound and Vibration, 209, 882-888

16. Kim J.-O., LeE K.-S., LeE J.-W., 2008, Beam stability on an elastic foundation subjected to distributed follower force, Journal of Mechanical Science and Technology, 22, 2386-2392

17. Kim N.I., 2010, Dynamic stability behavior of damped laminated beam subjected to uniformly distributed subtangential forces, Composite Structures, 92, 2768-2780

18. Kim N.I., 2011, Divergence and flutter instability of damped laminated beams subjected to a triangular distribution of nonconservative forces, Advances in Structural Engineering, 14, 1075-1091 
19. Kirillov O.N., 2013, Nonconservative Stability Problems of Modern Physics, Walter de Gruyter $\mathrm{GmbH}$, Berlin/Boston

20. Krowiak A., 2008, Methods based on the differential quadrature in vibration analysis of plates, Journal of Theoretical and Applied Mechanics, 46, 123-139

21. Kumar A., Srivasta A.K., 1986, Stability of thin rectangular elastic plates under a follower force, Mechanics Research Communications, 13, 165-168

22. Langthjem M.A., Sugiyama Y., 2000, Dynamic stability of viscoelastic beam under follower forces, Journal of Sound and Vibration 238, 809-851

23. Leipholz, H.H.E., 1975, An extremum principle for the buckling problem of the clamped-clamped rod subjected to tangential follower forces, Mechanics Research Communications, 2, 119-123

24. Leipholz H.H.E., 1978, Stability of a rectangular simply supported plate subjected to nonincreasing tangential follower forces, ASME Journal of Applied Mechanics, 45, 223-224

25. Leipholz H.H.E., Bhalla K., 1977, On the solution of the stability problem of elastic rods subjected to triangularly distributed tangential follower forces, Ingenieur-Archiv, 46, 115-124

26. Leipholz, H.H.E., Pfendt F., 1982, On the stability of rectangular completely supported plates with uncoupled boundary conditions subjected to uniformly distributed follower forces, Computer Methods in Applied Mechanics Engineering, 30, 19-52

27. Leipholz H.H.E., Pfendt F., 1983, Application of extended equations of Galerkin to stability problems of rectangular plates with free edges and subjected to uniformly distributed follower forces, Computer Methods in Applied Mechanics and Engineering, 37, 341-365

28. Marzani A., Potapov V.D., 1999, On the stability of a nonlinear viscoelastic rod subjected to a longitudinal force in the form of a random stationary process, Mechanics of Time-Dependent Materials, 2, 335-349

29. Przybylski J., 1999, Instability regions of a prestressed compound column subjected to a follower force, Journal of Theoretical and Applied Mechanics, 37, 148-162

30. Robinson M.T.A., 2013, Nonlinear vibration of 2D viscoelastic plate subjected to tangential follower force, Engineering Mechanics, 20, 59-74

31. Robinson M.T.A., Adali S., 2016, Nonconservative stability of viscoelastic rectangular plates with free edges under uniformly distributed follower force, International Journal of Mechanical Sciences, 107, 150-159

32. RuAN M., WANG Z.-M., WANG Y., 2012, Dynamic stability of functionally graded materials skew plates subjected to uniformly distributed tangential follower forces, Journal of Vibration and Control, 18, 913-923

33. Ryu B.J., Sugiyama Y., Yim K.B., Lee G.S., 2000, Dynamic stability of an elastically restrained column subjected to triangularly distributed subtangential forces, Computers and Structures, 76, 611-619

34. Sugiyama Y., Kawagoe H., 1975, Vibration and stability of elastic columns under the combined action of uniformly distributed vertical and tangential forces, Journal of Sound and Vibration, 38, $341-355$

35. Sugiyama Y., Mladenov K.A., 1983, Vibration and stability of elastic columns subjected to triangularly distributed sub-tangential forces, Journal of Sound and Vibration, 88, 447-457

36. Tomski L., Szmidla J., 2004, Vibration and stability of a column subjected to the generalised load by a force directed towards the pole, Journal of Theoretical and Applied Mechanics, 42, 163-193

37. Tomski L., Uzny S., 2008, Free vibration and the stability of a geometrically non-linear column loaded by a follower force directed towards the positive pole, International Journal of Solids and Structures, 45, 87-112

38. Tomski L., Uzny S., 2013a, Free vibrations and stability of a new slender system subjected to a conservative or non-conservative load, ASCE Journal of Engineering Mechanics, 139, 1133-1148 
39. Tomski L., Uzny S., 2013b, Vibrations and stability of a column subjected to the specific load realized by circular elements of heads, Mechanics and Mechanical Engineering, 17, 197-206

40. Torki M.E., Kazemi M.T., Haddadpour H., Mahmoudkhani S., 2014a, Dynamic stability of cantilevered functionally graded cylindrical shells under axial follower forces, Thin-Walled Structures, 79, 138-146

41. Torki M.E., Kazemi M.T., Reddy J.N., Haddadpoud H., Mahmoudkani S., 2014b, Dynamic stability of functionally graded cantilever cylindrical shells under distributed axial follower forces, Journal of Sound and Vibration, 333, 801-817

42. WAng Y., WAng Z., ZU L., 2013, Stability of viscoelastic rectangular plate with a piezoelectric layer subjected to follower force, Archive of Applied Mechanics, 83, 495-507

43. WANG Z.M., Ji Y.Z., 1992, The dynamic stability of rectangular plates under the action of tangential follower force, Journal of Vibration Engineering, 5, 78-83

44. Wang Z.M., Zhou Y.-F., Wang Y., 2007, Dynamic stability of non-conservative viscoelastic rectangular plate, Journal of Sound and Vibration, 307, 250-264

45. WANG Z., WANG Y., Guo X., 2009, Dynamic stability of linearly varying thickness viscoelastic rectangular plate with crack and subjected to tangential follower force, Applied Acoustics, 70, 845-856

46. Zhou Y.-F., WAng Z.-M., 2014, Exact solutions for the stability of viscoelastic rectangular plate subjected to tangential follower force, Archive of Applied Mechanics, 84, 1081-1089

47. Zhuo R.H., Fen S.Z., 2005, Dynamic stability of viscoelastic beam under follower force, $A S C E$ Journal of Engineering Mechanics, 22, 26-30

48. Zuo Q.H., Shreyer H.L., 1996, Flutter and divergence instability of nonconservative beams and plates, International Journal of Solids and Structures, 33, 1355-1367 